\title{
Estimation of Traffic Management and Road Safety
}

\author{
K Praveen Kumar1)
}

\begin{abstract}
Cooperation between vehicles facilitates traffic management, road safety and movie applications. Cooperation, however, needs trust within the validity of the received info. during this paper, we have a tendency to tackle the challenge of firmly exchanging parking spot availableness info. Trust is crucial so as to support the choice of whether or not the querying vehicle ought to regarding suppose deem trust admit accept have confidence have faith in place confidence in the received info about free parking spots near its destination and therefore ignore alternative doubtless free spots on the means. Therefore, we have a tendency to propose Parking Communities, which offer a distributed and dynamic suggests that to determine sure teams of vehicles serving to one another to firmly notice parking in their individual community space. Our approach relies on superior progressive cryptography and signature algorithms in addition as a well-understood mathematical trust rating model. This approach permits end-to-end encrypted request-response communications together with geocast and might be used as AN overlay to existing conveyance networking technologies. we offer a comprehensive comparison with alternative security architectures and simulation results showing the practicability of our approach.
\end{abstract}

KEYWORDS: Geocast, Communication, Networking, Progressive.

\section{EXISTING SYSTEM}

This approach permits end-to-end encrypted request-response ommunications together with geocast and might be used as AN overlay to existing conveyance networking technologies. we offer a comprehensive comparison with alternative security architectures and simulation results showing the practicability of our approach.We analyze the protocol compared to existing solutions in Section vi, which might conjointly serve for equalisation the implementation tradeoffs of Parking Communities. we offer simulation leads to Section. The paper concludes in Section.

\section{EXISTING SYSTEM ALGORITHMS}

In addition to the $\mathrm{DH}$ key agreement supported the separate power downside, there conjointly exist error correction code variants, that need a smaller key size ensuing in less energy, memory, and information measure consumption. DH-based key agreement protocols square measure designed for synchronous communications as against the asynchronous Elliptic Curve Integrated cryptography theme (ECIES).

\section{PROPOSED SYSTEM}

\footnotetext{
${ }^{1}$ Department of Information technology VFSTR University, Vadlamudi, Guntur. praveen.kazipeta.kumar@gmail.com
} 
pseudonym certificates for namelessness and placement privacy. we have a tendency to provides a elaborated analysis of attack situations and describe our implementation of the projected security design in IBR-DTN, AN open supply RFC 5050 implementation. we have a tendency to more give a comprehensiveanalysis in terms of a comparative analysis with alternative key and trust management protocols and simulation results. The remainder of this paper is structured as follows. Section discusses connected add the sector of key and trust management in conveyance networks. The projected Parking Community thought is introduced in Section. Attack situations on Parking Communities and their mitigations square measure bestowed in Section. Section describes a first implementation in AN overlay network supported IBR-DTN.

\title{
PROPOSED SYSTEM ALGORITHMS
}

Encryption and Signature algorithmic rule This section introduces our extensions to the Bundle Security Protocol and discusses the protection background of the used algorithms.

\begin{abstract}
ADVANTAGES
additionally to the prediction accuracy, another advantage of our methodology is its high potency of QoS prediction. this means that our methodology is a lot of ascendable than ancient CF strategies once applied to large-scale service recommender systems. this means that our methodology is a lot of ascendable than ancient CF strategies once applied to large-scale service recommender systems. the explanation is that, in most cases we will limit similar neighbor looking to atiny low set of users (or internet services), particularly once $\mathrm{K}$ is little.
\end{abstract}

\section{MODULE DESCRIPTION}

internet services

cooperative Filtering (CF)

internet Service Recommendation

Incorporating QoS Variation into User and repair Similarity activity

Incorporating Locations of Users and Services into Similar Neighbor choice

User location info handler

Service location info handler

User-based QoS prediction

Service-based QoS prediction

Hybrid QoS prediction

Recommender

\section{Location illustration}


Location info Acquisition

Location scientific discipline

\section{Web services}

CF-based internet service recommendation aims to predict missing QoS (Quality-of-Service) values of internet services. With the prevalence of Service-Oriented design (SOA), a lot of and a lot of net applications square measure made by composing internet services. As a consequence, variety of internet services has redoubled speedily over the last decade. Collaborative Filtering (CF) is wide utilized to rec-ommend top quality internet services to service users. supported the actual fact that a service user might solely have in-voked atiny low variety of internet services, CF-based internet service recommendation technique focuses on predicting missing QoS values of internet services for the user.

\section{Collaborative Filtering $(\mathrm{CF})$}

cooperative filtering may be a methodology of constructing automatic predictions (filtering) concerning the interests of a user by aggregation preferences or style info from several users (collaborating) CF techniques is usually rotten into 2 categories: model-based and memory-based [12],[13]. Memory-based CF is additionally named neighborhood-based CF. counting on whether or not user neighborhood or item neighborhood is taken into account, neighborhood-based CF will more be classified into user-based and item primarily based. For example, mistreatment the temporal context, a travel recommender system would offer a vacation recommendation in winter terribly completely different from the one provided in summer. They incontestible that incorporating[1] discourse info in essence would improve each the effectiveness and therefore the potency of a recommender system.

\section{Web Service Recommendation}

varied recommendation techniques have recently been applied to internet service recommendation, like the content- primarily based link prediction-based. Their argued that, for each try of ac-tive user and target internet service, each the QoS expertise of the users like the active user and therefore the QoS values of the services like the target service is em-ployed for QoS prediction. However, these previous ap-proaches did not exploit the characteristics of QoS within the similarity computation. supported the standard CF approaches, many en-hanced strategies are projected to boost the pre-diction accuracy. this can be probable if the net services square measure deployed in a very high performance Cloud atmosphere. If the QoS is sweet enough (as during this instance), atiny low variation of QoS values over all users is probably going to be ob-served. Some internet services might have a really poor QoS for all users. 


\section{Incorporating QoS Variation into User and repair Similarity activity}

Previous QoS prediction strategies assume that the co-invoked internet services have equal contribution weights once computing similarity between 2 users. we have a tendency to argue that the personalised characteristics (e.g., QoS variation) of each internet services and users ought to be incorporated into activity the similarity among users and services. internet service QoS factors, like time interval, avail-ability and responsibleness, square measure typically user-dependent. From completely different internet services, we will derive completely different personal-ized characteristics[2], supported their QoS values, as perceived by a range of users. Some internet services might have a really sensible QoS for all users. For example, the availabil-ity is often 100 percent. this can be probable if the net services square measure deployed in a very high performance Cloud atmosphere. If the QoS is sweet enough (as during this instance), atiny low variation of QoS values over all users is probably going to be ob-served. Some internet services might have a really poor QoS for all users. for instance, the supply is often below five hundredth. this can be probable if the net services square measure deployed in a very network atmosphere with poor performance and information measure. These internet services also are seemingly to own little variation of QoS values over completely different users. several alternative internet services might have a comparatively giant variation of QoS over completely different users. for instance, the supply varies from five hundredth to 100 percent for various users. These internet services square measure thought-about to be user-sensitive[3]. the subsequent example explains why internet services with completely different QoS variations may contribute otherwise once computing the similarity between service users. User location info handler: This module obtains location info of a user together with the network and therefore the country in step with the user's science address. It conjointly provides support for economical user-querying supported location. Service location info handler: This handler acquires extra location info of internet services in step with either their URLs or science addresses. the placement info includes the network and therefore the country during which the net service square measure placed. It conjointly provides functionalities for supporting economical locationbased internet service question. User-based QoS prediction: when an explicit variety of comparable users square measure known for the active user, this operate aggregates the QoS values they perceived on track internet services, and predicts the missing QoS values for the active user. Service-based QoS prediction: when an explicit variety of comparable services square measure known for a target internet service, this operate aggregates their QoS values to predict the missing QoS values for the active user

Hybrid QoS prediction: This operate combines the userbased QoS prediction and therefore the service-based QoS prediction results, creating final QoS predictions. The cold-start downside and data-sparsity downside in QoS predictions also are

self-addressed during this module 
Recommender: when predicting missing QoS values for all candidate internet services, this operate recommends internet services with best QoS to the active user LOCATION info illustration, ACQUISITION, AND process

This section discusses the way to represent, acquire, and pro-cess location info of each internet services and ser-vice users, that lays a necessary foundation for imple-menting our location-aware internet service recommendation methodology.

\section{Location illustration}

We represent a user's location as a triple ( $\mathrm{IPu}, \mathrm{ASNu}$, CountryIDu), wherever scienceu denotes the IP address of the user, ASNu denotes the ID of the Autonomous System (AS)1 that IPu belongs to, and CountryIDu denotes the ID of the country that IPu belongs to. Typically, a rustic has several ASs ANd an AS is at intervals one country solely. the net consists of thousands of ASs that inter- connected with one another. Generally speaking, intra-AS traffic is way higher than inter-AS traffic concerning transmission performance, like re-sponse time [4]. Also, traffic between neighboring ASs is healthier than that between distant ASs. Therefore, the Inter-net AS-level topology has been wide wont to live the space between net users [4]. Note that users placed within the same AS aren't perpetually geographically shut, and the other way around. for instance, 2 users placed within the same town could also be at intervals completely different ASs. Therefore, notwithstanding 2 users square measure placed within the same town, they will look distant on the net if they're at intervals completely different ASs. This explains why we decide AS rather than alternative geographic positions, like latitude and great circle, to represent a user's location.

\section{Location info}

Acquisition deed the placement info of each internet services and repair users is simply done. as a result of the users' science addresses square measure already noted, to get full location in-formation of a user, we have a tendency to solely got to establish each the AS and therefore the country during which he's placed in step with his science address. variety of services and databases[5] square measure on the market for this purpose (e.g. the Whois search service2). during this work, we have a tendency to accomplished the science to AS mapping and science to country mapping mistreatment the GeoLite Autonomous System variety Database3. The information is updated each month, guaranteeing that neither the science to AS mapping nor the science to country mapping are going to be outdated.

\section{SIMILARITY COMPUTATION AND SIMILAR NEIGHBOR choice}

during this section, we have a tendency to 1 st formally outline notations for the convenience of 
describing our methodology and algorithms. we have a tendency to then gift a weighted PCC for computing similarity between each users and internet services, that takes their personal QoS characteristics into thought.

Finally, we have a tendency to discuss incorporating locations of each users and internet services into the similar neighbor choice.

\section{Similar Neighbor choice}

Similar neighbor choice may be a vital step of CF. choosing the neighbors right like the active user is important for correct missing worth prediction. In standard user-based $\mathrm{CF}$, the Top-K similar neighbor choice algorithmic rule is commonly utilized [8]. It selects $\mathrm{K}$ users that square measure most like the active user as his/her neighbors. Similarly, the Top-K similar neighbor choice algorithmic rule is utilized to pick $\mathrm{K}$ internet services that square measure most like the target internet service. There square measure many issues concerned, however, once applying the Top-K similar neighbor choice algorithmic rule to internet service recommendation. Firstly, in follow, some service users have either few similar users or no similar users owing to the information meagreness. ancient Top- $K$ algorithms ignore this downside and still opt for the highest $\mathrm{K}$ most ones. as a result of the ensuing neighbors aren't really like the target user (service), doing this can impair the prediction accuracy. Therefore, removing those neighbors from the highest $\mathrm{K}$ similar neighbor set is healthier if the similarity is not any over zero. Secondly, as antecedently mentioned, internet service users might happen to understand similar QoS values on a couple of internet services. however they're not extremely similar. Considering the location-relatedness of internet service QoS, we have a tendency to incorporate the locations of each users and internet services into similar neighbor choice.

\section{User-based QoS worth Prediction}

In this subdivision, we have a tendency to gift a user-based location-aware CF methodology, named as ULACF. ancient user-based CF strategies typically adopt for missing worth predictions. This equation, however, could also be inaccurate for internet service QoS worth prediction for the subsequent reasons. internet service QoS factors like time interval and output, that square measure objective parameters and their values vary mostly. In distinction, user ratings utilized by ancient recommender systems square measure subjective and their values square measure comparatively fastened [2]. Therefore, predicting QoS values supported the typical QoS values perceived by the active user (i.e., $r(u)$ ) is blemished. Moreover, Eq. (9) doesn't distinguish native and remote users that square measure like the active user. Intuitively, given 2 users that have a similar calculable similarity degree to the target user, the user nearer to the target user ought to be placed a lot of confidence in QoS prediction than the opposite. Item-based QoS worth Prediction In this subdivision, we have a tendency to gift AN item-based locationaware CF methodology, named as 
ILACF. supported the similar thought as ULACF's, we use Eq. to work out the expected QoS worth for a service supported the QoS values of its similar services

\section{Integrating QoS Predictions}

Due to the meagreness of the user-item matrix, to create the missing worth prediction as correct as potential, it's higher to totally explore the data of comparable users in addition as similar services. Therefore, we have a tendency to develop a hybrid location- aware $\mathrm{CF}$, named as HLACF, that integrated the user- based QoS prediction with the item-based QoS prediction. the subsequent four cases are going to be thought-about in desegregation QoS predictions

\section{Conclusion:}

Cooperation, however, wants trust among the validity of the received information. throughout this paper, we've an inclination to tackle the challenge of firmly exchanging parking spot handiness information. Trust is crucial therefore on support the selection of whether or not or not the querying vehicle need to relating to suppose view as trust admit settle for confidence in have faith in situ confidence within the received information about free parking spots close to its destination and so ignore various likely free spots on the means that. Therefore, we've an inclination to propose Parking Communities, which supply a distributed and dynamic suggests that to work out positive groups of vehicles serving to 1 another to firmly notice parking in their individual community house. Our approach depends on superior progressive cryptography and signature algorithms additionally as a well-understood mathematical trust rating model. This approach permits end-to-end encrypted request-response communications in conjunction with geocast and may well be used as associate degree overlay to existing conveyance networking technologies.

\section{REFERENCES:}

[1.]Brown, Eric (13 September 2016). "Who Needs the Internet of Things?". Linux.com. Retrieved 23 October 2016.

[2.]Brown, Eric (20 September 2016). "21 Open Source Projects for IoT". Linux.com. Retrieved 23 October 2016.

[3.]"Internet of Things Global Standards Initiative". ITU. Retrieved 26 June 2015.

[4.]"Internet of Things: Science Fiction or Business Fact?" Harvard Business Review. November 2014. Retrieved 23 October 2016.

[5.]Vermesan,Ovidiu;Friess,Peter(2013).InternetofThings:ConvergingTechnologiesforSmartEnvironmentsandIntegratedEcosy stemsAalborg, Denmark: River Publishers. ISBN 978-87-92982-96-4.

[6.]"An Introduction to the Internet of Things (IoT)"Cisco.com. San Francisco, California: Lopez Research. November 2013. Retrieved 23 October 2016. 